\title{
SENSITIVITY OF THE "INTERMEDIATE POINT" IN THE MEAN VALUE THEOREM: AN APPROACH VIA THE LEGENDRE-FENCHEL TRANSFORMATION
}

\author{
JEAN-BAPtiste HiRIART-URRUTY ${ }^{1}$
}

\begin{abstract}
We study the sensitivity, essentially the differentiability, of the so-called "intermediate point" $c$ in the classical mean value theorem $\frac{f(b)-f(a)}{b-a}=f^{\prime}(c)$; we provide the expression of its gradient $\nabla c(d, d)$, thus giving the asymptotic behavior of $c(a, b)$ when both $a$ and $b$ tend to the same point $d$. Under appropriate mild conditions on $f$, this result is "universal" in the sense that it does not depend on the point $d$ or the function $f$. The key tool to get at this result turns out to be the LEGENDRE-FENCHEL transformation for convex functions.
\end{abstract}

\section{INTRODUCTION}

The basic mean value theorem (MVT for short), also called LAGRANGE's MVT, is certainly one of the best known results in Calculus. Let us recall it. Given a differentiable function $f: I \rightarrow \mathbb{R}$ on some open interval $I$, for any $a<b$ in $I$, there exists $c \in(a, b)$ such that

$$
\frac{f(b)-f(a)}{b-a}=f^{\prime}(c)
$$

We call (1) the MVT relation. The MVT theorem is mainly an existence result, it does not say more on this "intermediate point $c$ "..., especially if it is unique or not. In this note, we precisely consider the situation where such a $c$ is unique for any $a<b$, and study the sensitivity of $c$ as a function of both variables $a$ and $b$ (its continuity, and differentiability).

Since one does not know much about such $c, c$ sometimes has the qualifier "mysterious". Here are some preliminary comments about it:

- The intermediate point(s) $c$ could be anywhere between $a$ an $b$. We can say more on their location if we know more about the generating function $f$; a typical example is when $f$ is a polynomial function of degree $n$ at most (see [10, Section 6.5] for that).

- When $f^{\prime}$ is strictly monotone on $(0,+\infty), c(a, b)=\left(f^{\prime}\right)^{-1}\left[\frac{f(b)-f(a)}{b-a}\right]$ is a way of defining "means" of $a>0$ and $b>0$, but not all means are defined in such a way (cf. [8]). For example, with the help of the generating function $x>0 \mapsto f(x)=\exp x$, one gets at $c(a, b)=\ln \left(\frac{e^{b}-e^{a}}{b-a}\right)$.

\footnotetext{
1 Institut de Mathématiques, Université Paul Sabatier

118 route de Narbonne, Toulouse (France).

jbhu@math.univ-toulouse.fr
}

(C) The authors. Published by EDP Sciences, SMAI 2021

This is an Open Access article distributed under the terms of the Creative Commons Attribution License (https://creativecommons.org/licenses/by/4.0), which permits unrestricted use, distribution, and reproduction in any medium, provided the original work is properly cited. 
- One could expect for $c$ some homogeneity properties like: for the same function $f$, the intermediate point $c(t a, t b)$ on the dilated interval $(t a, t b)$, with $t>0$, is the dilated version of the intermediate point on $(a, b)$, that is $t c(a, b)$. This is not true, it is even exceptional. For example, $c(a, b)$ just above does not satisfy this property. According to a deep and nice result by S. GolaB and S. LoJAsiewicz [3], this happens only for three classes of functions (we restrict ourselves to strictly convex or strictly concave functions $f$ on $(0,+\infty))$ : With $\alpha \neq 0$,

$$
\begin{aligned}
& f: \quad x \in(0,+\infty) \mapsto f(x)=\alpha x \ln x+\beta x+\gamma ; \\
& f: \quad x \in(0,+\infty) \mapsto f(x)=\alpha \ln x+\beta x+\gamma ; \\
& f \quad: \quad x \in(0,+\infty) \mapsto f(x)=\alpha x^{p}+\beta x+\gamma(\text { where } p \neq 0 \text { and } p \neq 1) .
\end{aligned}
$$

This is the occasion to propose various positively homogeneous means of the type $c(a, b)$ :

- If $f(x)=x^{2}$, then $c(a, b)=\frac{a+b}{2}$ (arithmetic mean of $a$ and $b$ ); if $f(x)=1 / x$, then $c(a, b)=\sqrt{a b}$ (geometric mean of $a$ and $b$ ). But there is no function $f$ for which the corresponding $c(a, b)$ would be the harmonic mean $\frac{2 a b}{a+b}$ of $a$ and $b$. Let us give a hint for that (from [8, pages $\left.682-683\right]$ ). According to what has been seen above, the (positively homogeneous) harmonic mean could only come from a function of the type $f: x>0 \mapsto f(x)=x^{p}$ (where $p \neq 0$ and $p \neq 1$ ). But this is impossible. Indeed, if that was the case for some power $p$, one would have:

$$
\begin{aligned}
& \frac{f(2)-f(1)}{2-1}=p[H(1,2)]^{p-1}, \\
& \frac{f(3)-f(1)}{3-1}=p[H(1,3)]^{p-1},
\end{aligned}
$$

where $H(a, b)$ stands for the harmonic mean of $a$ and $b$. The first equation, that is $\left(\frac{2^{p}-1}{p}\right)^{\frac{1}{p-1}}=\frac{4}{3}$, leads to $p=-4.14457 \ldots$; the second one, that is $\left(\frac{3^{p}-1}{2 p}\right)^{\frac{1}{p-1}}=\frac{3}{2}$, leads to $p=-4.36363 \ldots$.

- The logarithmic mean $c(a, b)=\frac{b-a}{\ln b-\ln a}$ comes from the generating function $\ln x$; the so-called identric mean $c(a, b)=\left(\frac{b^{b}}{a^{a}}\right)^{1 /(b-a)} / e$ comes from the generating function $x \ln x$.

- We can play with the power $p$ of the function $x^{p}$ and get at some unusual (positively homogeneous) means of $a$ and $b$. To take some examples,

$$
\begin{aligned}
& c(a, b)=\left(\frac{\sqrt{a}+\sqrt{b}}{2}\right)^{2} \text { if } f(x)=\sqrt{x} ; c(a, b)=\left(\frac{2 a^{2} b^{2}}{a+b}\right)^{1 / 3} \text { if } f(x)=\frac{1}{x^{2}} ; c(a, b)=\sqrt{\frac{a^{2}+a b+b^{2}}{3}} \text { if } \\
& f(x)=x^{3} ; c(a, b)=\left(\frac{\sqrt{a} \sqrt{b}(\sqrt{a}+\sqrt{b})}{2}\right)^{2 / 3} \text { if } f(x)=\frac{1}{\sqrt{x}} ; \text { etc. }
\end{aligned}
$$

For what we have to explore in this note, there is no loss of generality in assuming that $f$ is defined on the whole of $\mathbb{R}$, that is to say $I=\mathbb{R}$.

Let us list here some qualitative properties related to this intermediate point $c$ (see [7] for proofs):

- Such a point $c$ is unique for any $a<b$, if and only if either $f$ or $-f$ is strictly convex on $\mathbb{R}$. That militates for our choice thereafter: $f: \mathbb{R} \rightarrow \mathbb{R}$ is a differentiable strictly convex function.

- Such a point $c$ can sometimes be expressed as a function of $a$ and $b$, in a closed form; it depends on how easily the strictly increasing function $f^{\prime}$ can be inverted (see some examples above). There is however a simple basic situation in that respect: If $f(x)=\frac{1}{2} \alpha x^{2}+\beta x+\gamma$, with $\alpha \neq 0$, then $c=\frac{a+b}{2}$ for all $a<b$. It is interesting to note that this property of $c$ characterizes quadratic functions [7]. In the same vein, given $p \in(0,1 / 2)$, it is impossible to have $c=\frac{p a+(1-p) b}{2}$ for all $a<b[7]$. 
From now on, we define the "intermediate point function" $c: \mathbb{R} \times \mathbb{R} \rightarrow \mathbb{R}$ as follows:

$$
\left\{\begin{array}{c}
\text { For } a \neq b, c(a, b) \text { is the unique } c \text { satisfying the MVT relation (1) } \\
c(d, d)=d \text { for all } d \in \mathbb{R} .
\end{array}\right.
$$

The questions we address in this paper are:

- (The easiest one). Is $c$ a continuous function of $a$ and $b$ ?

- (A more elaborate one). Is $c$ a differentiable function of $a$ and $b$ ? If so, what is the gradient vector of $c$ at any point $(d, d)$ lying on the critical diagonal line of $\mathbb{R} \times \mathbb{R}$ ?

We answer both questions, delineating the appropriate (minimal) general assumptions on $f$ for that.

\section{BASIC TOOLS FROM ANALYSIS}

\subsection{The extended difference quotient}

Given a differentiable strictly convex function $f: \mathbb{R} \rightarrow \mathbb{R}$, we define the extended difference quotient function $q: \mathbb{R} \times \mathbb{R} \rightarrow \mathbb{R}$ as follows:

$$
\left\{\begin{array}{c}
\text { For } a \neq b, q(a, b)=\frac{f(b)-f(a)}{b-a} \\
q(d, d)=f^{\prime}(d) \text { for all } d \in \mathbb{R} .
\end{array}\right.
$$

Property: $q$ is a continuous function on $\mathbb{R} \times \mathbb{R}$.

This is a standard result in differential calculus. Let us still provide a short proof for the convenience of the reader.

The only nontrivial point to check is that $q$ is continuous at $(d, d)$.

Since $f$ is convex and differentiable, it is $C^{1}$. Therefore, $f$ is (so-called) strictly differentiable at $d$, so that

$$
\begin{aligned}
f(a)-f(b) & =f^{\prime}(d)(a-b)+(a-b) \varphi(a, b), \text { with } \\
\lim _{\substack{a \rightarrow d \\
b \rightarrow d}} \varphi(a, b) & =0 .
\end{aligned}
$$

But, $q(a, b)-q(d, d)=\varphi(a, b)$. Thus $\lim _{\substack{a \rightarrow d \\ b \rightarrow d}} q(a, b)=q(d, d)$.

We can even go further on the properties of $q$, assuming more about the function $f$.

Property: Assume that $f$ is $C^{2}$. Then $q$ is a continuously differentiable function on $\mathbb{R} \times \mathbb{R}$, with

$$
\nabla q(d, d)=\frac{1}{2}\left(\begin{array}{c}
f^{\prime \prime}(d) \\
f^{\prime \prime}(d)
\end{array}\right)
$$

This is again an exercise in differential calculus. Let us prove it quickly.

At a point $(a, b)$ with $a \neq b$, we clearly have:

$$
\begin{aligned}
& \frac{\partial q}{\partial a}(a, b)=\frac{f(b)-f(a)-(b-a) f^{\prime}(a)}{(b-a)^{2}} \\
& \frac{\partial q}{\partial b}(a, b)=\frac{f(a)-f(b)-(a-b) f^{\prime}(b)}{(b-a)^{2}}
\end{aligned}
$$

Following second-order TAYLOR-LAGRANGE expansions of $f$ at $a$ and at $b$, there exist $\xi_{1}$ and $\xi_{2}$ in $(a, b)$ such that $\frac{\partial q}{\partial a}(a, b)=\frac{1}{2} f^{\prime \prime}\left(\xi_{1}\right)$ and $\frac{\partial q}{\partial b}(a, b)=\frac{1}{2} f^{\prime \prime}\left(\xi_{2}\right)$. By the assumed continuity of $f^{\prime \prime}$, we therefore get:

$$
\lim _{\substack{a \rightarrow d \\ b \rightarrow d}} \frac{\partial q}{\partial a}(a, b)=\frac{1}{2} f^{\prime \prime}(d) ; \lim _{\substack{a \rightarrow d \\ b \rightarrow d}} \frac{\partial q}{\partial b}(a, b)=\frac{1}{2} f^{\prime \prime}(d) .
$$

Thus, $q$ is differentiable at $(d, d)$ and $(3)$ holds. 


\subsection{The LegendRe-FenCHeL transformation}

Since the given function $f$ is (strictly) convex, it is natural to evoke the LEGENDRE-FENCHEL transform for any property of $f$. Indeed, for our purposes its use turns out to be incredibly efficient.

The LEGENDRE-FEnCHEL transform (or conjugate) $f^{*}$ of $f$ is defined as follows:

$$
s \in \mathbb{R} \mapsto f^{*}(s)=\sup _{x \in \mathbb{R}}(s x-f(x)) .
$$

We use $s$ to denote the variable because, in the geometrical interpretation of definition (4), $s$ is a slope. We denote by $I^{*}$ the interval on which the supremum in (4) is finite. When $f^{\prime}$ is invertible, which is our case (the strict convexity of $f$ implies that $f^{\prime}$ is strictly increasing), another way of looking at $f^{*}$ is:

$$
f^{*}(s)=s \cdot\left(f^{\prime}\right)^{-1}(s)-f\left[\left(f^{\prime}\right)^{-1}(s)\right] .
$$

This is the usual form of the so-called LEGENDRE transform of $f$, whether $f$ is convex or not. In terms of derivatives, the relation between $f$ and $f^{*}$ is simple: the derivative function of $f^{*}$ is the inverse of the derivative function of $f$ :

$$
f^{\prime}(x)=s \Leftrightarrow\left(f^{*}\right)^{\prime}(s)=x
$$

This property could even help obtain an antiderivative (or primitive function) of $f$, see an example below (Example 2.4). To get familiar with the transformation $f \rightsquigarrow f^{*}$, it is best to consider examples, which we do below.

Example 2.1. Let $f(x)=\frac{1}{2} \alpha x^{2}+\beta x+\gamma$, with $\alpha>0$. Then

$$
f^{*}(s)=\frac{s^{2}}{2 \alpha}-\frac{\beta}{\alpha} s+\frac{\beta^{2}}{2 \alpha}-\gamma .
$$

Here $I^{*}=\mathbb{R}$ and $f^{*}$ is of the same kind (i.e., quadratic) as $f$.

Example 2.2. Let $f(x)=\exp x$. Then, for $s \geqslant 0$,

$$
f^{*}(s)=s \ln s-s .
$$

As usual, we understand that $0 \ln 0=0$. In this example, $I^{*}=[0,+\infty)$. This is certainly one of the simplest examples to illustrate the relationship (6) between the derivatives of $f$ and $f^{*}: f^{\prime}(x)=\exp x$ for all $x$, while $\left(f^{*}\right)^{\prime}(s)=\ln s$ for all $s>0$.

Example 2.3. Let $f(x)=\sqrt{1+x^{2}}$. Then, for $|s| \leqslant 1$,

$$
f^{*}(s)=-\sqrt{1-x^{2}}
$$

Here $I^{*}=[-1,1]$.

Example 2.4. Let $f(x)=\cosh x$. Then, a simple calculation of $f^{*}(s)$ defined in (4) leads to

$$
\begin{aligned}
f^{*}(s) & =s \cdot \sinh ^{-1} s-\cosh \left(\sinh ^{-1} s\right) \\
& =s \cdot \sinh ^{-1} s-\sqrt{1+s^{2}} .
\end{aligned}
$$

Since $f^{\prime}=\sinh$ and its inverse $\left(f^{\prime}\right)^{-1}$ is the derivative of $f^{*}$, the above calculation is an alternate way of determining an antiderivative (or primitive) function of $\sinh ^{-1}$ : it is the function given in (7).

All these examples illustrate formulas (5) and (6); we will even go further, in due time, with a relationship between second derivatives of $f$ and $f^{*}$. 
The properties of the LEGENDRE-FENCHEL transformation are well-known and used in convex analysis (see for example [11, section 26] or [5, chapter E]). We list below those we need for our purposes. Recall that we have assumed that $f: \mathbb{R} \rightarrow \mathbb{R}$ is a strictly convex differentiable function. We then have:

(1) $I^{*}$ is an interval with a nonempty interior, and $f^{*}$ is differentiable on $\operatorname{int}\left(I^{*}\right)$.

(2) $\lim _{k \rightarrow \infty}\left|f^{\prime}\left(x_{k}\right)\right|=+\infty$ whenever $\left(x_{k}\right)$ is a sequence in $\operatorname{int}\left(I^{*}\right)$ converging to an endpoint of $\operatorname{int}\left(I^{*}\right)$.

Note that $(2)$ holds vacuously when $I^{*}$ is the whole of $\mathbb{R}$.

Said in different words: $\operatorname{int}\left(I^{*}\right)$ is parameterized by the injective function $f^{\prime}$, and for $s \in \operatorname{int}\left(I^{*}\right), f^{*}(s)=$ $s . x-f(x)$, where $x$ is the unique solution of the equation $s=f^{\prime}(x)$.

Examples 2.1-2.4 illustrate perfectly well the above-displayed properties of $f^{*}$.

We complement the relationship $\left(f^{*}\right)^{\prime}=\left(f^{\prime}\right)^{-1}$ with one on the second-derivatives [2, 12, 13]:

(3) Suppose $f$ is $C^{2}$ with $f^{\prime \prime}>0$ on $\mathbb{R}$. Then so is $f^{*}$ on the interior of $I^{*}$, with:

$$
\left(f^{*}\right)^{\prime \prime}(s)=\frac{1}{f^{\prime \prime}(x)} \text {, where } x \text { is the unique solution of } s=f^{\prime}(x) \text {. }
$$

Let us quickly illustrate the property above with the functions displayed in Example 2.3. We have

$$
\begin{aligned}
f^{\prime}(x) & =\frac{x}{\sqrt{1+x^{2}}}, f^{\prime \prime}(x)=\frac{1}{\left(1+x^{2}\right)^{\frac{3}{2}}}, \\
\text { while }\left(f^{*}\right)^{\prime}(s) & =\frac{1}{\sqrt{1-s^{2}}},\left(f^{*}\right)^{\prime \prime}(s)=\frac{1}{\left(1-s^{2}\right)^{\frac{3}{2}}} .
\end{aligned}
$$

Indeed, $s=x / \sqrt{1+x^{2}}, x \in \mathbb{R}$, is a way of parameterizing the interior of $I^{*}(=(-1,1))$, and, for all $s=$ $x / \sqrt{1+x^{2}}$, we indeed have $\left(f^{*}\right)^{\prime \prime}(s)=1 / f^{\prime \prime}(x)$.

\section{The MAin RESUlts}

We begin with the following key observation on the function $c$ defined in (2):

$$
c=\left(f^{*}\right)^{\prime} \circ q .
$$

This is indeed true for $a \neq b$, since by definition

$$
\begin{aligned}
c(a, b) & =\left(f^{\prime}\right)^{-1}\left(\frac{f(b)-f(a)}{b-a}\right) \\
& =\left(f^{*}\right)^{\prime}\left(\frac{f(b)-f(a)}{b-a}\right)=\left(\left(f^{*}\right)^{\prime} \circ q\right)(a, b),
\end{aligned}
$$

but also at diagonal points $(d, d)$ since

$$
\begin{aligned}
c(d, d) & =d=\left(f^{*}\right)^{\prime}\left(f^{\prime}(d)\right) \\
& =\left(\left(f^{*}\right)^{\prime} \circ q\right)(d, d) .
\end{aligned}
$$

It now remains to scroll the results, everything has been prepared for that.

Theorem 3.1 (continuity). Let $f: \mathbb{R} \rightarrow \mathbb{R}$ be strictly convex and differentiable. For $(a, b)$ in $\mathbb{R} \times \mathbb{R}$, let $c(a, b)$ be the intermediate point in the $M V T$, as defined in (2). Then $c$ is a continuous function on $\mathbb{R} \times \mathbb{R}$.

Proof. We have seen in Section 2.1 that the extended difference quotient $q$ is a continuous function from $\mathbb{R} \times \mathbb{R}$ into $\operatorname{int}\left(I^{*}\right)$. Moreover, $\left(f^{*}\right)^{\prime}: \operatorname{int}\left(I^{*}\right) \rightarrow \mathbb{R}$, the derivative function of a differentiable convex function, is continuous. Hence, $c$ which is nothing but $\left(f^{*}\right)^{\prime} \circ q$ (see $\left.(9)\right)$, is a continuous function. 
The result of Theorem 3.1 is not completely new, it appears in the following alternative form in the literature (in $[3,4,9]$ for example): Assuming that $f^{\prime}$ is strictly monotone, $\theta(a, h)$ is defined as the unique parameter in $(0,1)$ for which $(f(a+h)-f(a)) / h=f^{\prime}(a+\theta(a, h) h)$; then $\theta$ is a continuous function of $a$ and $h$.

Theorem 3.2 (differentiability). Let $f: \mathbb{R} \rightarrow \mathbb{R}$ be twice continuously differentiable with $f^{\prime \prime}>0$. For $(a, b)$ in $\mathbb{R} \times \mathbb{R}$, let $c(a, b)$ be the intermediate point in the $M V T$, as defined in (2). Then $c$ is a continuously differentiable function on $\mathbb{R} \times \mathbb{R}$, with

$$
\nabla c(d, d)=\left(\begin{array}{c}
1 / 2 \\
1 / 2
\end{array}\right) \text { for all }(d, d) \text { in } \mathbb{R} \times \mathbb{R}
$$

Proof. Again, $c=\left(f^{*}\right)^{\prime} \circ q$, resulting from the composition of two continuously differentiable functions, is itself continuously differentiable.

As for $\nabla c(d, d)$, it suffices to apply the chain rule, with (3) and (8) as ingredients:

$$
\begin{aligned}
\nabla c(d, d) & =\left(f^{*}\right)^{\prime \prime}\left(f^{\prime}(d)\right) \cdot \frac{1}{2}\left(\begin{array}{l}
f^{\prime \prime}(d) \\
f^{\prime \prime}(d)
\end{array}\right) \\
& =\frac{1}{f^{\prime \prime}(d)} \cdot \frac{1}{2}\left(\begin{array}{c}
f^{\prime \prime}(d) \\
f^{\prime \prime}(d)
\end{array}\right)=\left(\begin{array}{c}
1 / 2 \\
1 / 2
\end{array}\right) .
\end{aligned}
$$

\section{Remarks.}

- By applying Theorem 3.2 partially, we recover the following result on the behavior of the intermediate point in the MVT (see [1] for example, but it was known long before): assume that $f$ is twice continuously differentiable in a neighborhood of a fixed $a$, with $f^{\prime \prime}(a) \neq 0$, then the intermediate point $c$ in the MVT property has the following asymptotic behavior

$$
\lim _{b \rightarrow a} \frac{c-a}{b-a}=\frac{1}{2}
$$

Indeed, one can choose $f^{\prime \prime}(a)>0$ without loss of generality, and extend $f$ to the whole of $\mathbb{R}$ so as to satisfy the assumptions in Theorem 3.2. Then, use the fact that $\frac{\partial c}{\partial b}(a, a)=\frac{1}{2}$.

- The "perfect" or asymptotic case is when $f$ is quadratic, $f(x)=\frac{1}{2} \alpha x^{2}+\beta x+\gamma$, with $\alpha>0$ (see Example 2.1). In that case, $c(a, b)=\frac{a+b}{2}$ for all $(a, b)$ in $\mathbb{R} \times \mathbb{R}$.

What Theorem 3.2 entails is that, under appropriate assumptions on $f$, say $f^{\prime \prime}(d)>0, c(a, b) \sim \frac{a+b}{2}$ when $a$ and $b$ are close to a same point $d$.

Theorem 3.2 also explains, a posteriori, what we have evoked at the beginning: given $p \in(0,1 / 2)$, it is impossible to have $c=\frac{p a+(1-p) b}{2}$ for all $a<b$, except for affine functions $f$ (for which $f^{\prime \prime}=0$ ).

- One cannot weaken the assumptions in Theorem 3.2 by just requiring $f$ to be twice continuously differentiable and strictly convex. For example, when considering the function $x \in \mathbb{R} \mapsto f(x)=x^{4}$, its second derivative is $f^{\prime \prime}(x)=12 x^{2}$, which vanishes only at 0 . Then, for the intermediate point, whose explicit expression here is $c(a, b)=\left(\frac{a^{3}+a^{2} b+a b^{2}+b^{3}}{4}\right)^{1 / 3}$, we have

$$
\frac{\partial c}{\partial a}(0,0)=\frac{\partial c}{\partial b}(0,0)=\frac{1}{4^{1 / 3}}
$$

Final comments.

- As any differentiability result, Theorem 3.2 measures the "sensitivity" of $c(a, b)$ with respect to changes to the point $(d, d)$. The fact that $\frac{\partial c}{\partial a}(d, d)=\frac{\partial c}{\partial b}(d, d)$ just expresses the symmetry of this sensitivity of $c$ with respect to the two variables $a$ and $b$; this is indeed a reasonable and expected result. 
- Note that the value of $\nabla c(d, d)$ does not change with $d$, and is independent of the function $f$ (satisfying the assumptions in Theorem 3.2)! It is a kind of "universal result", as it sometimes happens in mathematics.

As a by-product of this result, we note that all the means of the form $c(a, b)$ (see the beginning of the paper) have the same asymptotic behavior, $c(a, b) \sim \frac{a+b}{2}$, when $a$ and $b$ are close to a same point $d$.

We would like to thank the two referees for their careful reading of the submitted manuscript and the subsequent remarks.

\section{REFERENCES}

[1] A.G. Azpeitia, On the Lagrange remainder of the Taylor formula, American Math. Monthly Vol. 89, ${ }^{\circ} 5$ (1982), $311-312$.

[2] J.-P. Crouzeix, A relationship between the second derivatives of a convex function and of its conjugate. Math. Programming 13 (1977), $364-365$.

[3] S. Golab and S. Lojasiewicz, Un théorème sur la valeur moyenne $\theta$ dans la formule des accroissements finis. Annales Polonici Mathematici 3 (1956), $118-125$.

[4] J.V. Conçalves, Sur l'inconnue $\theta$ du théorème des accroissements finis. Portugaliae Mathematica 2 (1941), 121 - 128.

[5] J.-B. Hiriart-Urruty and C. Lemaréchal. Fundamentals of convex analysis. Grundlehren Text Editions Springer (2001).

[6] J.-B. Hiriart-Urruty, Mathematical Tapas. Vol. 1 (for Undergraduates). Springer Undergraduate Mathematics Series (2016).

[7] J.-B. Hiriart-Urruty, Mean value theorems and convexity: an example of cross-fertilization of two mathematical items. Elemente Der Mathematik, Vol. 75, $\mathrm{N}^{\circ} 2(2020), 69-79$.

[8] M.E. MaYs, Functions which parametrize means. The American Mathematical Monthly 90, n 10 (1983), 677 - 683.

[9] B. Morduknovich, Qualitative relation of functions $f$ and $\theta$ in Lagrange theorem. Vestnik of Belorussian State University of V.I. Lenin, Series $1, \mathrm{n}^{\circ} 1$ (1970), $91-92$ (in Russian).

[10] Q.I. Rahman and G. Schmeisser, Analytic Theory of Polynomials. Clarendon Press, Oxford (2002).

[11] R.T. Rockafellar, Convex Analysis. Vol. 28 of Princeton Math. Series, Princeton University Press (1970).

[12] R.T. Rockafellar, Higher derivatives of conjugate convex functions. The International Journal of Applied Analysis, vol. 1, $\mathrm{n}^{\circ} 1,(1977), 41-42$.

[13] A. SeEger, Second derivatives of a convex function and of its Legendre-Fenchel transformate. SIAM J. Optimization Vol. 2, $\mathrm{n}^{\circ} 3$ (1992), $405-424$. 